\title{
Durable complete response after afatinib and crizotinib in an advanced non-small cell lung cancer patient with EGFR L861Q mutation and acquired MET amplification: a case report
}

\author{
Yaling Long ${ }^{1 \#}$, Kai Zhang ${ }^{2 \#}$, Yanying $\mathrm{Li}^{1}$, Min Yu ${ }^{1}$, Jiao Zhu ${ }^{1}$, Meijuan Huang ${ }^{1}$ \\ ${ }^{1}$ Department of Thoracic Oncology, Cancer Center, ${ }^{2}$ Department of Radiology, West China Hospital, Medical School, Sichuan University, Chengdu, \\ China \\ \#These authors contributed equally to this work. \\ Correspondence to: Meijuan Huang. Department of Thoracic Oncology, Cancer Center, West China Hospital, Medical School, Sichuan University, \\ Chengdu, China. Email: hmj107@163.com.
}

\begin{abstract}
Epidermal growth factor receptor (EGFR) L861Q mutation is a non-classical mutation, with a low incidence, poor response, and uncertain resistance mechanisms when treated by an EGFR tyrosine kinase inhibitor (EGFR-TKI). The liver is one of the most common distant organs to metastasize in nonsmall cell lung cancer (NSCLC), and achieving complete remission treatment for the liver is difficult. In this report, a patient was diagnosed with advanced lung adenocarcinoma harboring the EGFR L861Q mutation and responded well to afatinib for 16 months. Complete response and partial response (PR) appeared in the liver metastasis and primary lesion respectively. Following this, afatinib plus crizotinib overcame the acquired resistance of MET amplification and brought about the complete remission of the liver for 10 months. Interestingly, the liver remission endured for 22 months and persisted even when the disease progressed and the EGFR T790M mutation emerged. To our knowledge, this is the first time that afatinib induced longterm liver remission in a patient with an EGFR non-classical mutation, and in whom crizotinib with afatinib proved to be a reliable treatment for overcoming MET amplification resistance with an EGFR non-classical mutation. This precise and individualized gene-based treatment significantly prolonged the survival time of this stage IV case with brain metastases yielding 26 months of progression-free survival (PFS) time and more than 3 years of overall survival time.
\end{abstract}

Keywords: Non-small cell lung cancer (NSCLC); EGFR L861Q mutation; liver metastasis; MET amplification; EGFR T790M mutation; case report

Submitted Nov 12, 2019. Accepted for publication May 27, 2020.

doi: 10.21037/apm-19-482

View this article at: http://dx.doi.org/10.21037/apm-19-482

\section{Introduction}

Nearly $22.5 \%$ and $52.5 \%$ of Western and Asian patients with non-small cell lung cancer (NSCLC), respectively, have positive epidermal growth factor receptor (EGFR) mutations (1). However, there is a subset of patients (10$15 \%$ ) with some type of non-classical mutations (L861Q, G719X, S768I) who show limited response to firstgeneration EGFR tyrosine kinase inhibitors (EGFR-TKIs). Liver metastasis is a predictive factor for poor response in lung cancer $(2,3)$. Single MET amplification and EGFR
T790M mutation have been confirmed as mechanisms of acquired resistance to EGFR non-classical mutations (4). Here, we report a patient who was diagnosed with advanced lung adenocarcinoma harboring EGFR nonclassical mutation L861Q and who achieved tumor control and complete continuous response in liver metastasis with afatinib. More importantly, afatinib plus crizotinib overcame the resistance of MET amplification. Remission of the liver metastasis continued even after the EGFR T790M mutation emerged and the disease progressed. We 
present the following case in accordance with the CARE reporting checklist.

\section{Case presentation}

A 54-year-old non-smoking Asian female who was healthy previously presented in September 2016 with dry cough. No history of cancer was identified in her family. Computed tomography (CT) scan of the chest showed an enlarged mass in the right hilum with enlarged right hilar and mediastinal lymph nodes (Figure $1 A, B$ ). CT scan on the abdomen (Figure $1 C$ ) revealed a metastatic tumor in the right lobe of the liver. Magnetic resonance imaging (MRI) of the brain found two metastases in the left frontal lobe and right parietal lobe. She was diagnosed with lung adenocarcinoma (cT4N3M1c, stage IV) harboring EGFR exon 21 (L861Q) mutation by percutaneous lung biopsy and next-generation sequencing (NGS).

First-line chemotherapy with pemetrexed plus carboplatin started in November 2016. Partial response (PR) was achieved in the first 4 cycles of chemotherapy, and stereotactic radiotherapy was applied for the brain metastases. However, both the pulmonary lesion and the liver metastasis progressed in the next 2 cycles of maintenance chemotherapy with pemetrexed in April 2017 (Figure 1D,E,F). The patient then accepted afatinib (40 mg orally once daily) on April 13, 2017, as the secondline treatment. The pulmonary lesion and liver metastasis effectively shrank and PR was achieved after treatment by afatinib for 28 days (Figure $1 G, H, I$ ). On October 8, 2017, the primary lesion and brain metastases sustained $\mathrm{PR}$ and the liver metastasis could no longer be detected (Figure 17,K,L). Multiple pulmonary metastases appeared in August 2018. The progression-free survival (PFS) of secondline treatment was nearly 16 months. Tissue rebiopsy of her emerging lesion in the left lung detected EGFR L861Q mutation at 56.64\% and MET amplification with 9.54 copy numbers by NGS. Taking her gene mutations into consideration, we started the patient on a combined administration of afatinib and crizotinib in September 2018 (Figure 1M,N,O). Pulmonary lesions regained PR (Figure 1P). Pulmonary lesions eventually grew in July 2019 (Figure 1Q). The PFS of third-line treatment was nearly 10 months. Tissue re-biopsy of her growing right lung lesion detected EGFR L861Q mutation at 74.72\% and EGFR T790M mutation at $52.41 \%$ by NGS. The patient then received osimertinib as a fourth-line treatment beginning in August 2019. Of significant note, PR of the brain metastases and CR of liver metastasis persisted for 22 months until the latest follow-up day on August 9, 2019 (Figure $1 R$ ). The summary of the patient's treatment is shown in Figure 2.

The patient reported a rash, hand-foot syndrome, liver dysfunction, and diarrhea over the duration of treatment with afatinib. However, adverse events of grade 2 severity (according to CTCAE 4.0) only included diarrhea and elevated hepatic enzymes. The combination of afatinib with crizotinib did not increase the incidence and severity of adverse events. Written informed consent was obtained from the patient for publication of this manuscript and any accompanying images.

\section{Discussion}

EGFR mutations occur within exons 18-21, nearly 10-15\% are non-classical mutations that have a lower objective response rate and shorter PFS than patients with common mutations $(5,6)$, especially those treated by first-generation EGFR-TKIs $(7,8)$. Limited evidence has demonstrated the efficacy of afatinib and osimertinib in those patients with non-classical mutations (9). To our knowledge, there are few, if any, reports of long-lasting CR achieved in liver metastasis along with successful combination therapy overcoming the acquired resistance in non-classical mutation.

The effective response in the patient's liver metastasis with non-classical EGFR mutation might be associated with tumor heterogeneity and the mechanism of afatinib. The contribution of genetic variability and tumor heterogeneity to EGFR-TKI resistance in lung cancer has been confirmed by several studies $(10,11)$. In the present case, the resistance of MET amplification was tested in the lung while CR persisted in the liver. Afatinib is an irreversible ErbB family blocker that inhibits the kinase activity of all ErbB family members including Erb-B2 receptor tyrosine kinase 3 (ErbB3, HER3) (12). There is a study demonstrating increased hepatocyte growth factor (HGF) expression when cancer invades and grows in the liver (2). Considering that HGF has been suspected of activating the downstream pathway of tumorigenesis through ErbB3 (13), it is reasonable to speculate that the broad spectrum of activity across ErbB family members and the enzymatically irreversible binding to active ErbB family receptors of the second-generation EGFR-TKI might result in effective control in liver metastasis.

Data on the acquired resistance mechanism of nonclassical EGFR mutations are limited. Amplification of 

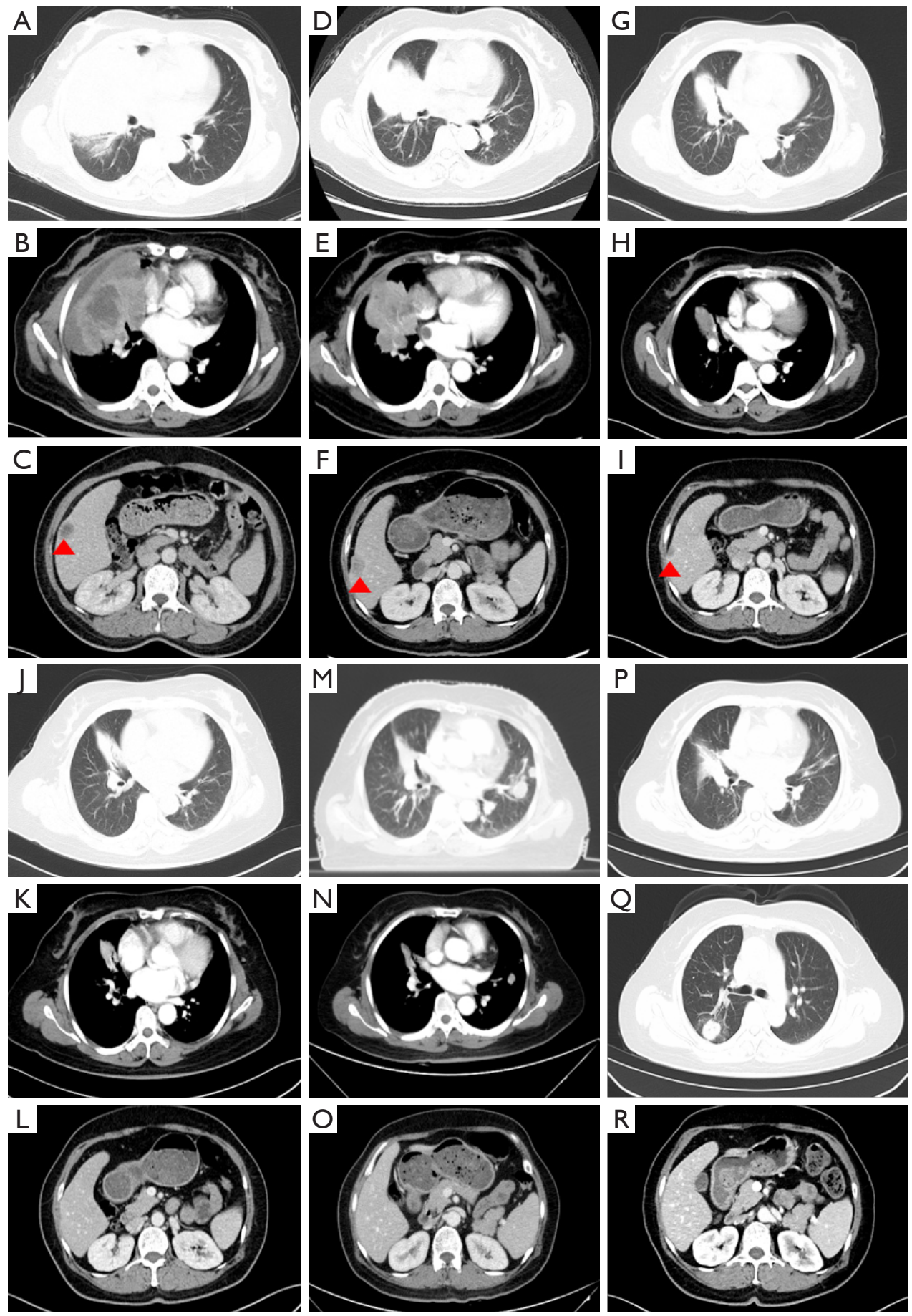

Figure 1 The changes in patient's CT images. (A,B,C) The baseline of lung lesion and liver metastasis in September 2016; (D,E,F) the lung lesion shrunk after 4 cycles of chemotherapy, but liver metastases progressed; (G,H,I) the lung lesion and liver metastasis achieved partial response after treatment by afatinib for 1 month; $(\mathrm{J}, \mathrm{K}, \mathrm{L})$ the lung lesion maintained partial response after treatment by afatinib for 6 months, and liver metastasis achieved complete response; (M,N,O) multiple metastases in the bilateral lung appeared after treatment by afatinib for 16 months, but liver metastasis sustained complete remission; $(\mathrm{P})$ partial response was regained in the pulmonary lesion treated by the combination of afatinib and crizotinib; (Q) the right lung lesions eventually grew after afatinib and crizotinib at 10 months; (R) complete remission of the liver metastasis was sustained as of the final follow-up. Arrowheads: the metastasis in the right lobe of the liver. 


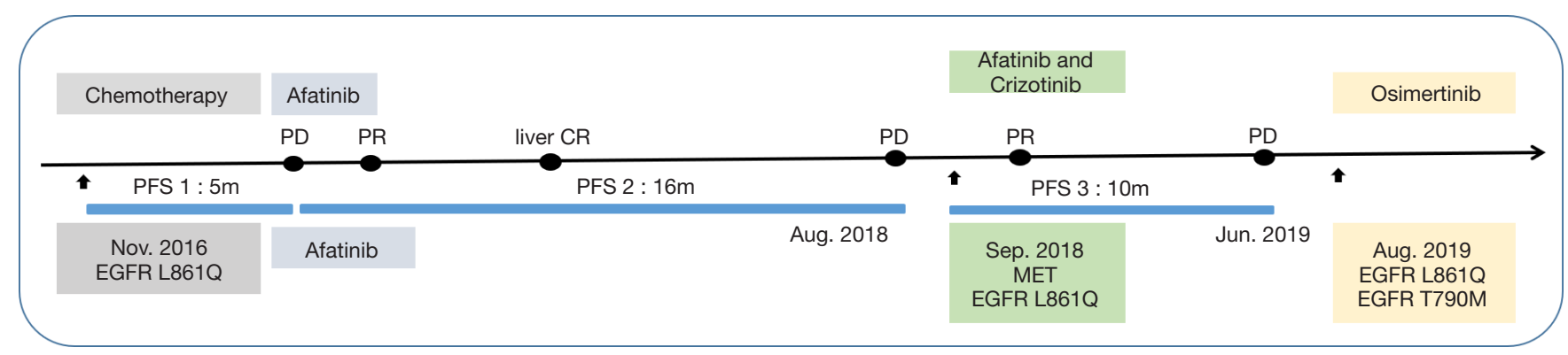

Figure 2 The summary of the patient's treatment.

the MET gene and EGFR T790M mutation account, respectively for $\sim 5 \%$ and $\sim 60 \%$ of EGFR-TKI resistance cases among classical EGFR mutation patients (4). The literature contains some case series and small sample studies of crizotinib with or without EGFR-TKI for acquired MET amplification in EGFR non-classical mutations resistant to first or third EGFR-TKIs obtaining a positive result (14-18). However, there is no report about combined secondgeneration drugs and MET inhibitor in patients with nonclassical EGFR mutations. It is the first time that the regimen above was proved to be both effective and tolerable in patient with EGFR non-classical mutation. Nevertheless, this case report only acted as a reference. The unanswered questions about the mechanism need further research.

\section{Conclusions}

We report here an extremely advanced NSCLC with EGFR non-classical mutation case that achieved an enduring CR in liver metastasis and long-term survival time based on precise and individual treatment. Although the exact mechanisms involved remain unclear, this case report provides a reference for the treatment and acquired resistance of an analogous patient. Meanwhile, for the first time, the combination of afatinib with crizotinib to overcome the resistance of MET amplification and EGFR non-classical mutation was confirmed to be a safe and reliable therapeutic strategy.

\section{Acknowledgments}

Funding: This work was supported by the Sichuan Provincial Research Foundation for Basic Research (2018SZ0023).

\section{Footnote}

Conflicts of Interest: All authors have completed the ICMJE uniform disclosure form (available at http://dx.doi. org/10.21037/apm-19-482). The authors have no conflicts of interest to declare.

Ethical Statement: The authors are accountable for all aspects of the work in ensuring that questions related to the accuracy or integrity of any part of the work are appropriately investigated and resolved. All procedures performed in studies involving human participants were in accordance with the Helsinki Declaration (as revised in 2013). All clinical investigations were approved by the West China Hospital of Sichuan University. Written informed consent was obtained from the patient for publication of this manuscript and any accompanying images.

Open Access Statement: This is an Open Access article distributed in accordance with the Creative Commons Attribution-NonCommercial-NoDerivs 4.0 International License (CC BY-NC-ND 4.0), which permits the noncommercial replication and distribution of the article with the strict proviso that no changes or edits are made and the original work is properly cited (including links to both the formal publication through the relevant DOI and the license). See: https://creativecommons.org/licenses/by-nc-nd/4.0/.

\section{References}

1. Vargas AJ, Harris CC. Biomarker development in the precision medicine era: lung cancer as a case study. Nat Rev Cancer 2016;16:525-37. 
2. He Y, Wang Y, Zhang S, et al. Hepatic metastasis is a poor predictive marker for erlotinib in lung adenocarcinoma. Med Hypotheses 2016;94:20-2.

3. Shi P, Oh Y'T, Zhang G, et al. Met gene amplification and protein hyperactivation is a mechanism of resistance to both first and third generation EGFR inhibitors in lung cancer treatment. Cancer Lett 2016;380:494-504.

4. Westover D, Zugazagoitia J, Cho BC, et al. Mechanisms of acquired resistance to first- and second-generation EGFR tyrosine kinase inhibitors. Ann Oncol 2018;29:i10-9.

5. $\mathrm{Xu} \mathrm{J}$, Jin B, Chu T, et al. EGFR tyrosine kinase inhibitor (TKI) in patients with advanced non-small cell lung cancer (NSCLC) harboring uncommon EGFR mutations: A realworld study in China. Lung Cancer 2016;96:87-92.

6. Shen YC, Tseng GC, Tu CY, et al. Comparing the effects of afatinib with gefitinib or Erlotinib in patients with advanced-stage lung adenocarcinoma harboring nonclassical epidermal growth factor receptor mutations. Lung Cancer 2017;110:56-62.

7. Kobayashi Y, Mitsudomi T. Not all epidermal growth factor receptor mutations in lung cancer are created equal: Perspectives for individualized treatment strategy. Cancer Sci 2016;107:1179-86.

8. Li H, Wang C, Wang Z, et al. Efficacy and long-term survival of advanced lung adenocarcinoma patients with uncommon EGFR mutations treated with 1st generation EGFR-TKIs compared with chemotherapy as first-line therapy. Lung Cancer 2019;130:42-9.

9. Yang JC, Sequist LV, Geater SL, et al. Clinical activity of afatinib in patients with advanced non-small-cell lung cancer harbouring uncommon EGFR mutations: a combined post-hoc analysis of LUX-Lung 2, LUX-Lung 3, and LUX-Lung 6. Lancet Oncol 2015;16:830-8.

10. Hata A, Yoshioka H, Fujita S, et al. Complex mutations in the epidermal growth factor receptor gene in non-small cell lung cancer. J Thorac Oncol 2010;5:1524-8.

Cite this article as: Long Y, Zhang K, Li Y, Yu M, Zhu J, Huang M. Durable complete response after afatinib and crizotinib in an advanced non-small cell lung cancer patient with EGFR L861Q mutation and acquired MET amplification: a case report. Ann Palliat Med 2020;9(5):3609-3613. doi: 10.21037/ apm-19-482
11. Kobayashi S, Boggon TJ, Dayaram T, et al. EGFR mutation and resistance of non-small-cell lung cancer to gefitinib. N Engl J Med 2005;352:786-92.

12. Diz Taín P, Gonzalez AL, Garcia-Palomo A. Mechanism of action and preclinical development of afatinib. Med Clin (Barc) 2016;146 Suppl 1:7-11.

13. Engelman JA, Zejnullahu K, Mitsudomi T, et al. MET amplification leads to gefitinib resistance in lung cancer by activating ERBB3 signaling. Science 2007;316:1039-43.

14. Deng L, Kiedrowski LA, Ravera E, et al. Response to Dual Crizotinib and Osimertinib Treatment in a Lung Cancer Patient with MET Amplification Detected by Liquid Biopsy Who Acquired Secondary Resistance to EGFR Tyrosine Kinase Inhibition. J Thorac Oncol 2018;13:e169-72.

15. Kang J, Chen HJ, Wang Z, et al. Osimertinib and Cabozantinib Combinatorial Therapy in an EGFRMutant Lung Adenocarcinoma Patient with Multiple MET Secondary-Site Mutations after Resistance to Crizotinib. J Thorac Oncol 2018;13:e49-53.

16. Li YQ, Song SS, Jiang SH, et al. Combination therapy of erlotinib/crizotinib in a lung adenocarcinoma patient with primary EGFR mutation plus secondary MET amplification and a novel acquired crizotinib-resistant mutation MET G1108C. Ann Oncol 2017;28:2622-4.

17. Gainor JF, Niederst MJ, Lennerz JK, et al. Dramatic Response to Combination Erlotinib and Crizotinib in a Patient with Advanced, EGFR-Mutant Lung Cancer Harboring De Novo MET Amplification. J Thorac Oncol 2016;11:e83-5.

18. van Veggel B, de Langen AJ, Hashemi S, et al. Crizotinib treatment for patients with EGFR mutation positive NSCLC that acquire cMET amplification after EGFR TKI therapy results in short-lived and heterogeneous responses. Lung Cancer 2018;124:130-4. 